\title{
XMM-Newton observations of Seyfert galaxies from the Palomar spectroscopic survey: the X-ray absorption distribution
}

\begin{abstract}
A. Akylas and I. Georgantopoulos
Institute of Astronomy \& Astrophysics, National Observatory of Athens, I. Metaxa \& B. Pavlou, Penteli, 15236 Athens, Greece

Received 18 November 2008 / Accepted 16 March 2009

ABSTRACT

We present XMM-Newton spectral analysis of all 38 Seyfert galaxies from the Palomar spectroscopic sample of galaxies. These are found at distances of up to $67 \mathrm{Mpc}$ and cover the absorbed $2-10 \mathrm{keV}$ luminosity range $\sim 10^{38}-10^{43} \mathrm{erg} \mathrm{s}^{-1}$. Our aim is to determine the distribution of the X-ray absorption in the local Universe. Three of these are Compton-thick with column densities just above $10^{24} \mathrm{~cm}^{-2}$ and high equivalent width $\mathrm{FeK}_{\alpha}$ lines $(>700 \mathrm{eV})$. Five more sources have low values of the X-ray to [OIII] flux ratio suggesting that they could be associated with obscured nuclei. Their individual spectra show neither high absorbing columns nor flat spectral indices. However, their stacked spectrum reveals an absorbing column density of $N_{\mathrm{H}} \sim 10^{23} \mathrm{~cm}^{-2}$. Therefore the fraction of absorbed sources $\left(>10^{22} \mathrm{~cm}^{-2}\right)$ could be as high as $55 \pm 12 \%$. A number of Seyfert-2 appear to host unabsorbed nuclei. These are associated with low-luminosity sources $L_{\mathrm{X}}<3 \times 10^{41} \mathrm{erg} \mathrm{s}^{-1}$. Their stacked spectrum again shows no absorption while inspection of the Chandra images, where available, shows that contamination from nearby sources does not affect the XMM-Newton spectra in most cases. Nevertheless, such low luminosity sources are not contributing significantly to the X-ray background flux. When we consider only the brighter, $>10^{41} \mathrm{erg} \mathrm{s}^{-1}, 21$ sources, we find that the fraction of absorbed nuclei rises to $75 \pm 19 \%$ while that of Compton-thick sources to $15-20 \%$. The fraction of Compton-thick AGN is lower than that predicted by the X-ray background synthesis model in the same luminosity and redshift range.
\end{abstract}

Key words. surveys - X-rays: galaxies - X-rays: general

\section{Introduction}

The moderate to high redshift Universe has been probed at unparallelled depth with the most sensitive observations performed at X-ray wavelengths in the Chandra Deep fields. The Chandra 2Ms observations (Alexander et al. 2003; Luo et al. 2008) resolved about 80 per cent of the extragalactic X-ray light in the hard 2-10 keV band (see Brandt \& Hasinger 2005, for a review). These deep surveys find a sky density of 5000 sources per square degree, the vast majority of which are found to be AGN through optical spectroscopy (e.g. Barger et al. 2003). In contrast, the optical surveys for QSOs (e.g. the COMBO-17 survey) reach only a surface density about an order of magnitude lower (e.g. Wolf et al. 2003). This clearly demonstrates the power of $\mathrm{X}$-ray surveys for detecting AGN. This is because hard X-rays can penetrate large amounts of gas without suffering from significant absorption. Indeed detailed spectral analysis on X-ray selected AGN reveals large amount of obscuration (e.g. Akylas et al. 2006; Tozzi et al. 2006; Georgantopoulos et al. 2007). In particular, about two thirds of the X-ray sources, over all luminosities, present column densities higher than $10^{22} \mathrm{~cm}^{-2}$. These high absorbing columns are believed to originate in a molecular torus surrounding the nucleus.

However, even the efficient 2-10 keV X-ray surveys may be missing a fraction of highly obscured sources. This is because at very high obscuring column densities $\left(>10^{24} \mathrm{~cm}^{-2}\right.$, corresponding to an optical reddening of $\left.A_{\mathrm{V}}>100\right)$, the X-ray photons with energies between 2 and $10 \mathrm{keV}$ are absorbed. These are the
Compton-thick AGN (see Comastri 2004, for a review) where the Compton scattering on the bound electrons becomes significant. Despite the fact that Compton-thick AGN are abundant in our vicinity (e.g. NGC 1068, Circinus), only a few tens of Compton-thick sources have been identified from X-ray data (Comastri 2004). Although the population of Compton-thick sources remains elusive there is concrete evidence for its presence. The X-ray background synthesis models can explain the peak of the X-ray background at $30-40 \mathrm{keV}$, where most of its energy density lies, (Frontera et al. 2007; Churazov et al. 2007) only by invoking a large number of Compton-thick AGN (Gilli et al. 2007). Additional evidence for the presence of a Comptonthick population comes from the directly measured space density of black holes in the local Universe. It is found that this space density is a factor of two higher than that predicted from the X-ray luminosity function (Marconi et al. 2004). This immediately suggests that the X-ray luminosity function is missing an appreciable number of obscured AGN.

In recent years there have been many efforts to uncover heavily obscured and in particular Compton-thick AGN in the local Universe by examining IR or optically selected, [OIII], AGN samples. This is because both the IR and the narrow-line region originate beyond the obscuring region and thus represent an isotropic property of the AGN. Risaliti et al. (1999) examine the X-ray properties of a large sample of [OIII] selected Seyfert-2 galaxies whose X-ray spectra were available in the literature. They find a large fraction of Compton-thick sources (over half of their sample). Their estimates are complemented by 
more recent XMM-Newton observations of local AGN samples (Cappi et al. 2006; Panessa et al. 2006; Guainazzi et al. 2005). All these authors also claim a large Compton-thick AGN fraction exceeding 30 per cent of the Seyfert-2 population. The advent of the SWIFT and INTEGRAL missions which carry X-ray detectors with imaging capabilities (e.g. Barthelmy et al. 2005; Ubertini et al. 2003) in ultra-hard X-rays (15-200 keV) try to shed new light on the absorption properties of AGN in the local Universe. In principle, at these ultra-hard X-rays obscuration should play a negligible role, at least up to column densities as high as $10^{25} \mathrm{~cm}^{-2}$. However, because of the limited effective area the above surveys can provide $\mathrm{X}$-ray samples, down to very bright fluxes $10^{-11} \mathrm{erg} \mathrm{cm}^{-2} \mathrm{~s}^{-1}$, with limited quality spectra. Again XMM-Newton observations are often required to determine the column density in each source. Interestingly, these surveys find only a limited number of Compton-thick sources (Markwardt et al. 2005; Bassani et al. 2006; Malizia et al. 2007; Ajello 2008; Winter et al. 2008; Tueller et al. 2008; Sazonov et al. 2008).

Here, we present XMM-Newton observations of all 38 Seyfert galaxies in the Palomar spectroscopic sample of nearby galaxies (Ho et al. 1997). This is the largest complete optically selected AGN sample in the local Universe analyzed so far. 23 of the Seyfert galaxies presented here have already been discussed in previous works (e.g. Cappi et al. 2006). For 5 of them newer $X M M-N e w t o n$ observations are available and are presented here. The current work should provide the most unbiased census of the AGN column density distribution at low redshifts and luminosities.

\section{The sample}

The Seyfert sample used in this study is derived from the Palomar optical spectroscopic survey of nearby galaxies (Ho et al. 1995). This survey has taken high quality spectra of 486 bright $\left(B_{\mathrm{T}}<12.5 \mathrm{mag}\right)$, northern $\left(\delta>0^{\circ}\right)$ galaxies selected from the Revised Shapley-Ames Catalogue of Bright Galaxies (RSAC, Sandage \& Tammann 1979) and produced a comprehensive and homogeneous catalogue of nearby Seyfert galaxies. The catalogue is $100 \%$ complete to $B_{\mathrm{T}}<12.0 \mathrm{mag}$ and $80 \%$ complete to $B_{\mathrm{T}}<12.5 \mathrm{mag}$ (Sandage et al. 1981).

For the purpose of this work we consider all the Seyfert galaxies from the Palomar survey. Sources lying in-between the Seyfert-Liner or the Seyfert-Transient boundary have been excluded. Furthermore seven Seyfert galaxies (i.e. NGC 1068, NGC 1358, NGC 1667, NGC 2639, NGC 3185, NGC 4235, NGC 5548), which have been included in the Palomar survey for various reasons (see Ho et al. 1995), even though they did not satisfy the survey selection criteria, are also excluded.

There are 40 Seyfert galaxies comprising the optical sample. 9 sources are classified as type-1 (contains types $1,1.2,1.5$ ) and 31 as type-2 (contains types $1.8,1.9,2$ ) Seyfert galaxies. However NGC 4051, NGC 4395 and NGC 4639 which have been initially classified as Seyfert $1.2,1.8$ and 1 by Ho et al. (1997) has been re-classified as type-1.5, 1 and 1.5 respectively (see Cappi et al. 2006; Panessa et al. 2006; Baskin \& Laor 2008). Moreover NGC 185 which is classified as a Seyfert-2 may not contain an active nucleus since it presents line intensity ratios possibly produced by stellar processes (Ho \& Ulvestad 2001).

The main characteristics of these sources, taken from Ho et al. (1997), are listed in Table 1. Some galaxies listed here present $B_{\mathrm{T}}$ fainter than the formal limit of the Palomar survey. According to Ho et al. (1995) this discrepancy can be attributed to errors in the apparent magnitudes given in the RSAC.

\section{X-ray observations}

The X-ray data have been obtained with the EPIC (European Photon Imaging Cameras, Strüder et al. 2001; Turner et al. 2001) on board XMM-Newton. Thirty sources have been recovered from the XMM-Newton archive while the remaining ten objects (marked with a " $\star$ " in Table 2) have been observed by us during the Guest Observer program.

The $\log$ of all the XMM-Newton observations is shown in Table 2. The data have been analysed using the Scientific Analysis Software (SAS v.7.1). We produce event files for both the PN and the MOS observations using the EPCHAIN and EMCHAIN tasks of $S A S$ respectively. The event files are screened for high particle background periods. In our analysis we deal only with events corresponding to patterns $0-4$ for the PN and 0-12 for the MOS instruments.

The source spectra are extracted from circular regions with radius of 20 arcsec. This area encircles at least the 70 per cent of the all the X-ray photons at off-axis angles less than 10 arcmin. A ten times larger source-free area is used for the background estimation. The response and ancillary files are also produced using SAS tasks RMFGEN and ARFGEN respectively.

We note that 18 of the XMM-Newton observations presented here, coincide with these presented in Cappi et al. (2006). However we choose to re-analyze these common data-sets in order to present a uniform treatment of the sample.

\section{X-ray spectral analysis}

We investigate the X-ray properties of the sources in our sample by performing spectral fittings with XSPEC v.12.4 software package. 2 sources are excluded from the X-ray spectral analysis: the Seyfert-2 galaxy NGC185 for being undetected in the X-rays (see also Sect. 2), and the Seyfert-1.5 galaxy NGC 1275 which belongs to the Perseus cluster and whose X-ray image shows that its flux is heavily contaminated by diffuse emission.

The X-ray spectra are binned to give a minimum of 15 counts so Gaussian statistics can be applied. We fit the PN and the MOS data simultaneously in the $0.3-10 \mathrm{keV}$ range. However in some cases where a very complex behaviour is present we perform the spectral fits only in the $2-10 \mathrm{keV}$ band. These latter cases are denoted with an asterisk $(\star)$ in Table 3 .

The normalization parameters for each instrument are left free to vary within 5 per cent in respect to each other to account for the remaining calibration uncertainties.

We assume a standard power-law model with two absorption components ( $w a^{*} w a^{*} p o$ in XSPEC notation) to account for the source continuum emission. The first absorption column models the Galactic absorption. Its fixed values are obtained from Dickey \& Lockman (1990) and are listed in Table 3. The second absorption component represents the AGN intrinsic absorption and is left as a free parameter during the model fitting procedure. A Gaussian component has also been included to describe the $\mathrm{FeK}_{\alpha}$ emission line.

When the fitting procedure gives a rejection probability less than 90 per cent we accept the above "standard model". However when this simple parametrization is not sufficient to model the whole spectrum additional components are included. For example soft-excess emission and reflection are common features in the X-ray spectra of Seyfert galaxies and can be modeled using additional XSPEC models.

In particular we fit a second power-law model, with $\Gamma$ fixed to the direct component value, to account for the scattered X-ray radiation and/or a Raymond-Smith to model the contribution from 
Table 1. The sample.

\begin{tabular}{|c|c|c|c|c|c|}
\hline $\begin{array}{l}\text { Name } \\
\text { (1) }\end{array}$ & $\begin{array}{c}\alpha(\mathrm{J} 2000) \\
(2)\end{array}$ & $\begin{array}{c}\delta(\mathrm{J} 2000) \\
(3)\end{array}$ & $\begin{array}{c}B_{\mathrm{T}}(\mathrm{mag}) \\
(4)\end{array}$ & $\begin{array}{c}D(\mathrm{Mpc}) \\
(5)\end{array}$ & $\begin{array}{c}\text { Class } \\
(6)\end{array}$ \\
\hline NGC 0185 & 003857.40 & +482014.4 & 10.10 & 0.7 & $\mathrm{~S} 2$ \\
\hline NGC 0676 & 014857.38 & +05 5425.70 & 10.50 & 19.5 & S2: \\
\hline NGC 1058 & 024330.24 & +372027.20 & 11.83 & 9.1 & S2 \\
\hline NGC 1167 & 030142.40 & +351221.00 & 13.38 & 65.3 & S2 \\
\hline NGC 1275 & 031948.16 & +413042.38 & 12.64 & 70.1 & S1.5 \\
\hline NGC 2273 & 065008.71 & +605045.01 & 12.55 & 28.4 & S2 \\
\hline NGC 2655 & 085538.84 & +78 1325.20 & 10.96 & 24.4 & S2 \\
\hline NGC 3031 & 095533.17 & +690355.06 & 7.89 & 1.4 & S1.5 \\
\hline NGC 3079 & 100158.53 & +554050.10 & 11.54 & 20.4 & $\mathrm{~S} 2$ \\
\hline NGC 3147 & 101653.27 & +732402.40 & 11.43 & 40.9 & S2 \\
\hline NGC 3227 & 102330.58 & +195153.99 & 11.10 & 20.6 & S1.5 \\
\hline NGC 3254 & 102919.96 & +292929.60 & 12.41 & 23.6 & S2 \\
\hline NGC 3486 & 110024.10 & +285831.60 & 11.05 & 7.4 & S2 \\
\hline NGC 3516 & 110647.49 & +723406.80 & 12.50 & 38.9 & S1.2 \\
\hline NGC 3735 & 113557.49 & +703207.70 & 12.50 & 41.0 & S2: \\
\hline NGC 3941 & 115255.42 & +365910.50 & 11.25 & 18.9 & S2: \\
\hline NGC 3976 & 115557.35 & +064457.00 & 12.30 & 37.7 & S2: \\
\hline NGC 3982 & 115628.10 & +550730.50 & 11.78 & 17.0 & S1.9 \\
\hline NGC 4051 & 120309.61 & +443152.80 & 11.88 & 17.0 & S1.2 \\
\hline NGC 4138 & 120929.87 & +434106.00 & 12.16 & 17.0 & S1.9 \\
\hline NGC 4151 & 121032.57 & +392420.63 & 11.50 & 20.3 & S1.5 \\
\hline NGC 4168 & 121217.30 & +131217.9 & 12.11 & 16.8 & S1.9: \\
\hline NGC 4169 & 121218.93 & +291044.00 & 13.15 & 50.4 & $\mathrm{~S} 2$ \\
\hline NGC 4258 & 121857.54 & +471814.30 & 9.10 & 6.8 & S1.9 \\
\hline NGC 4378 & 122518.14 & +045531.60 & 12.63 & 35.1 & $\mathrm{~S} 2$ \\
\hline NGC 4388 & 122546.70 & +123940.92 & 11.76 & 16.8 & S1.9 \\
\hline NGC 4395 & 122548.93 & +333247.80 & 10.64 & 3.6 & S1.8 \\
\hline NGC 4472 & 122946.76 & +075959.90 & 9.37 & 16.8 & S2:: \\
\hline NGC 4477 & 123002.22 & +133811.30 & 11.38 & 16.8 & $\mathrm{~S} 2$ \\
\hline NGC 4501 & 123159.34 & +142513.40 & 10.36 & 16.8 & $\mathrm{~S} 2$ \\
\hline NGC 4565 & 123621.07 & +255913.50 & 10.42 & 9.7 & S1.9 \\
\hline NGC 4639 & 124252.51 & +131524.10 & 12.24 & 16.8 & $\mathrm{~S} 1$ \\
\hline NGC 4698 & 124822.98 & +082914.80 & 11.46 & 16.8 & S2 \\
\hline NGC 4725 & 125026.69 & +253002.30 & 10.11 & 12.4 & S2: \\
\hline NGC 5033 & 131327.52 & +363537.78 & 10.75 & 18.7 & S1.5 \\
\hline NGC 5194 & 132952.37 & +471140.80 & 8.96 & 7.7 & S2 \\
\hline NGC 5273 & 134208.33 & +353915.17 & 12.44 & 21.3 & S1.5 \\
\hline NGC 6951 & 203714.41 & +660619.70 & 11.64 & 24.1 & $\mathrm{~S} 2$ \\
\hline NGC 7479 & 230456.69 & +121923.20 & 11.60 & 32.4 & S1.9 \\
\hline NGC 7743 & 234421.44 & +095603.60 & 12.38 & 24.4 & $\mathrm{~S} 2$ \\
\hline
\end{tabular}

Column 1: Galaxy name. Columns 2 and 3: optical coordinates. Column 4: Total apparent $B$ magnitude taken from Ho et al. (1997). Column 5: source distance in Mpc from Ho et al. (1997). Column 6: optical classification from Ho et al. (1997). Quality ratings. are given by “:” and “::" for uncertain and highly uncertain classification.

diffuse emission in the host galaxy. A flattening of the spectrum is usually indicative of reflected radiation from the backside of the torus. The reflected radiation is modelled using the PEXRAV model (Magdziarz \& Zdziardski 1995). In order to accept the new component we apply the F-test criterion. If the addition of the new component significantly improves the fit at the 90 per cent confidence level, then it is accepted. Other characteristics such as ionized features could also be considered however once a reasonable fit is obtained (i.e. with rejection probability less than 90 per cent) we do not include additional components.

The best fit parameters for all the sources are reported in Table 3. The errors quoted correspond to the 90 per cent confidence level for one interesting parameter. We note here that some of the sources listed show a rather steep photon index. In many cases this happens because of the fixed value of the continuum power-law photon index to the photon index of the soft component (e.g. NGC 1358, NGC 3079, NGC 3735). When these parameters are untied the continuum power-law photon index becomes harder.
18 of the X-ray observations presented here have already been shown in Cappi et al. (2006). In most of these the results are in agreement. However some deviations also appear and are discussed below. In the cases of NGC 3486, NGC 3079, NGC 4051 and NGC 4388 the comparison is not straightforward since we use of a different spectral fitting model. When the same model is applied as a test, there is no significant difference in the results. In the cases of NGC 1058 and NGC 4725 our results show a steeper power-low photon index than that presented in Cappi et al. (2006). However we point out that the results are consistent within the 90 per cent confidence level.

The XMM-Newton X-ray spectra of our sources are presented in Fig. 6. For each object the upper panel shows the X-ray spectrum along with the model presented in Table 3 while the lower panel shows the residuals.

\section{X-ray absorption}

The spectral fitting results are presented in Table 3 . There are 8 type-1 Seyferts in our sample. Five of them show small amounts 
Table 2. Log of the XMM-Newton observations.

\begin{tabular}{|c|c|c|c|c|c|c|c|c|}
\hline \multirow[t]{2}{*}{ Name } & \multirow[t]{2}{*}{ Obs. Date } & \multirow[t]{2}{*}{ Obs. ID } & \multicolumn{3}{|c|}{ Exposure } & \multicolumn{3}{|c|}{ Filter } \\
\hline & & & PN & MOS1 & MOS2 & PN & MOS1 & MOS2 \\
\hline NGC 185 & 2004-01-09 & 0204790301 & - & 11393 & 11334 & closed & Medium & Medium \\
\hline NGC 676 & 2002-07-14 & 0112551501 & 17754 & 21127 & 21127 & Thick & Thin & Thin \\
\hline NGC 1058 & 2002-02-01 & 0112550201 & 12902 & 17019 & 17019 & Medium & Thin & Thin \\
\hline NGC $1167^{\star}$ & 2005-08-04 & 0301650101 & 9937 & 11448 & 11448 & Thin & Thin & Thin \\
\hline NGC 1275 & 2006-01-29 & 0305780101 & 119697 & 124801 & 124832 & Medium & Medium & Medium \\
\hline NGC 2273 & 2003-09-05 & 0140951001 & 11076 & 12709 & 12714 & Medium & Medium & Medium \\
\hline NGC $2655^{\star}$ & 2005-09-04 & 0301650301 & 9850 & 11564 & 11570 & Thin & Thin & Thin \\
\hline NGC 3031 & 2001-04-22 & 0111800101 & 129550 & 82790 & 83150 & Medium & Medium & Medium \\
\hline NGC 3079 & 2001-04-13 & 0110930201 & 20023 & 24661 & 24663 & Thin & Medium & Medium \\
\hline NGC 3147 & 2006-10-06 & 0405020601 & 14963 & 16923 & 16912 & Thin & Thin & Thin \\
\hline NGC 3227 & $2000-11-28$ & 0101040301 & 34734 & 37198 & 37201 & Medium & Medium & Medium \\
\hline NGC $3254^{\star}$ & $2005-10-31$ & 0301650401 & 9869 & 11489 & 11481 & Thin & Thin & Thin \\
\hline NGC 3486 & 2001-05-09 & 0112550101 & 9057 & 6398 & 6385 & Medium & Thin & Thin \\
\hline NGC 3516 & 2001-11-09 & 0107460701 & 12829 & 12901 & 12900 & Thin & Thin & Thin \\
\hline NGC $3735^{\star}$ & $2005-09-27$ & 0301650501 & 9312 & 16466 & 16471 & Thin & Thin & Thin \\
\hline NGC 3941 & 2001-05-09 & 0112551401 & 9389 & 14635 & 14331 & Medium & Thin & Thin \\
\hline NGC $3976^{\star}$ & 2006-06-16 & 0301651801 & 11313 & 13483 & 13598 & Thin & Thin & Thin \\
\hline NGC 3982 & 2004-06-15 & 0204651201 & 10197 & 11674 & 11679 & Thin & Thin & Thin \\
\hline NGC 4051 & $2002-11-22$ & 0157560101 & 49808 & 51510 & 51520 & Medium & Medium & Medium \\
\hline NGC 4138 & $2001-11-26$ & 0112551201 & 9999 & 14365 & 14365 & Medium & Thin & Thin \\
\hline NGC 4151 & $2003-05-26$ & 0143500201 & 18454 & 18602 & 18607 & Medium & Medium & Medium \\
\hline NGC 4168 & 2001-12-04 & 0112550501 & 18498 & 22864 & 22849 & Medium & Thin & Thin \\
\hline NGC 4169^ & 2006-06-20 & 0301651701 & 11068 & 12695 & 12701 & Thin & Thin & Thin \\
\hline NGC 4258 & 2006-11-17 & 0400560301 & 62607 & 64179 & 64184 & Medium & Medium & Medium \\
\hline NGC $4378^{\star}$ & 2006-01-08 & 0301650801 & 10963 & 12602 & 12604 & Thin & Thin & Thin \\
\hline NGC 4388 & $2002-12-12$ & 0110930701 & 8292 & 11666 & 11666 & Thin & Medium & Medium \\
\hline NGC 4395 & 2003-11-30 & 0142830101 & 10596 & 10942 & 10940 & Medium & Medium & Medium \\
\hline NGC 4472 & 2004-01-01 & 0200130101 & 89503 & 94179 & 94185 & Thin & Thin & Thin \\
\hline NGC 4477 & 2002-06-08 & 0112552101 & 9500 & 13501 & 13527 & Medium & Thin & Thin \\
\hline NGC 4501 & 2002-06-08 & 0112550801 & 2885 & 13387 & 13385 & Medium & Thin & Thin \\
\hline NGC 4565 & 2001-07-01 & 0112550301 & 10010 & 14261 & 14263 & Medium & Thin & Thin \\
\hline NGC 4639 & $2001-12-16$ & 0112551001 & 10000 & 14365 & 14265 & Medium & Thin & Thin \\
\hline NGC 4698 & $2001-12-17$ & 0112551101 & 11755 & 16112 & 16112 & Medium & Thin & Thin \\
\hline NGC 4725 & 2002-06-14 & 0112550401 & 13369 & 17244 & 17244 & Medium & Thin & Thin \\
\hline NGC 5033 & $2002-12-18$ & 0094360501 & 9999 & 11616 & 11614 & Medium & Medium & Medium \\
\hline NGC 5194 & 2003-01-15 & 0303420101 & 19047 & 49944 & 49351 & Thin & Thin & Thin \\
\hline NGC 5273 & 2002-06-14 & 0112551701 & 10392 & 16065 & 16094 & Medium & Thin & Thin \\
\hline NGC $6951^{\star}$ & 2005-06-05 & 0301651401 & 7951 & 9664 & 9669 & Thin & Thin & Thin \\
\hline NGC $7479^{\star}$ & 2005-06-05 & 0301651201 & 12315 & 15740 & 15750 & Thin & Thin & Thin \\
\hline NGC $7743^{\star}$ & $2005-06-15$ & 0301651001 & 11847 & 13283 & 13348 & Thin & Thin & Thin \\
\hline
\end{tabular}

Column 1: name of the Galaxy. Column 2: start observation date (UTC). Column 3: observation identifier. Columns 3-5: net exposure time for the EPIC instruments. Columns 6-8: applied filter. ${ }^{\star}$ Denotes sources observed during our Guest Observer program.

of absorption $\left(<10^{21} \mathrm{~cm}^{-2}\right)$ while the 3 Seyfert-1.5 sources (NGC 3227, NGC 3516, and NGC 4151) present a considerable amount of $N_{\mathrm{H}}\left(>10^{22} \mathrm{~cm}^{-2}\right)$. Our sample contains 30 Seyfert-2 galaxies. The column densities in this population vary from the Galactic to the Compton-thick limit $\left(N_{\mathrm{H}}>10^{24} \mathrm{~cm}^{-2}\right)$. However, the apparent number of significantly obscured sources is rather small. Only 12 out of 30 type- 2 sources present absorption greater than $10^{22} \mathrm{~cm}^{-2}$.

\subsection{Compton-thick sources}

The fraction of Compton-thick sources is more difficult to estimate. This is because the XMM-Newton effective area sharply decreases at energies higher than $6 \mathrm{keV}$. Given the limited $X M M-N e w t o n$ bandpass, which extends up to about $10 \mathrm{keV}$, we are not able to measure the absorption turnover for highly absorbed sources. A column density of $\sim 10^{24} \mathrm{~cm}^{-2}$ suppresses $90 \%$ of the flux in the $2-10 \mathrm{keV}$ band. Therefore, we can obtain a direct measurement of the obscuration only up to column densities reaching at most a few times $10^{24} \mathrm{~cm}^{-2}$. In the case of Compton-thick AGN the X-ray spectrum is dominated by scattered components from cold or warm material as well as an $\mathrm{FeK}_{\alpha}$ with high equivalent width (Matt et al. 2000). Then to unveil the presence of a Compton-thick nucleus we apply the following diagnostics.

- Flat X-ray spectrum $(\Gamma<1)$. This implies the presence of a strong reflection component, which intrinsically flattens the $\mathrm{X}$-ray spectrum at higher energies (e.g. Matt et al. 2000).

- High Equivalent Width of the $\mathrm{FeK}_{\alpha}$ line $(\sim 1 \mathrm{keV})$. This characteristic is consistent with a Compton-thick nucleus since then the line is measured against a much depressed continuum (Leahy \& Creighton 1993) or a pure reflected component.

- Low X-ray to optical flux ratio. Bassani et al. (1999) have showed that the $2-10 \mathrm{KeV}$ to the [OIII] $\lambda 5007$ flux ratio is very effective in the identification of Compton-thick sources. This is because the [OIII] $\lambda 5007$ (hereafter [OIII]) flux which 
Table 3. Spectral fits.

\begin{tabular}{|c|c|c|c|c|c|c|c|c|c|c|}
\hline $\begin{array}{l}\text { Name } \\
(1)\end{array}$ & $\begin{array}{c}N_{\mathrm{H}_{\mathrm{GAL}}} \\
(2)\end{array}$ & $\begin{array}{l}N_{\mathrm{H}} \\
(3)\end{array}$ & $\begin{array}{c}\Gamma_{\text {cont }} \\
(4) \\
\end{array}$ & $\begin{array}{l}k T \\
(5) \\
\end{array}$ & $\begin{array}{c}E W_{\mathrm{FeK}} \\
(6)\end{array}$ & $\begin{array}{l}F_{X} \\
(7)\end{array}$ & $\begin{array}{l}L_{\mathrm{X}} \\
(8) \\
\end{array}$ & $\begin{array}{c}f_{\text {scat }} \\
(9)\end{array}$ & $\begin{array}{l}f_{\text {refl }} \\
(10)\end{array}$ & $\begin{array}{l}\chi_{v}^{2} \\
(11)\end{array}$ \\
\hline NGC 676 & 4.7 & $<0.13$ & $2.10_{-0.27}^{+0.50}$ & - & - & 1.12 & 0.05 & - & - & $22.5 / 17$ \\
\hline NGC 1058 & 5.4 & $<0.53$ & $3.40_{-1.69}^{+2.65}$ & - & - & 0.24 & 0.02 & - & - & $31.1 / 34$ \\
\hline NGC 1167 & 9.8 & $0.32_{-0.41}^{+0.08}$ & $2.10_{-0.27}^{+0.50}$ & $0.33_{-0.04}^{+0.08}$ & - & 0.9 & 0.4 & - & - & $22.52 / 17$ \\
\hline NGC 2273 & 6.4 & $104.70_{-7.36}^{+15.90}$ & $1.84_{-0.05}^{+0.12}$ & $0.77_{-0.13}^{+0.08}$ & $1500_{-240}^{+303}$ & 89.4 & 8.5 & 0.01 & 1.35 & $125.1 / 114$ \\
\hline NGC 2655 & 2.2 & $42.69_{-530}^{+5.68}$ & $2.61_{-0.26}^{+0.30}$ & $0.73_{-0.26}^{+0.30}$ & 110.4 & 0.01 & 7.8 & - & - & $240.1 / 194$ \\
\hline NGC $3031^{\star}$ & 5.6 & $0.05_{-0.01}^{+0.02}$ & $1.91_{-0.08}^{+0.02}$ & $1.10_{-0.02}^{+0.02}$ & $53_{-14}^{+11}$ & 1164.7 & 0.3 & - & - & $1439.9 / 1350$ \\
\hline NGC 3079 & 0.9 & $201_{-89.8}^{+28.5}$ & $2.56_{-0.23}^{+0.02}$ & $0.84_{-0.03}^{+0.02}$ & $700_{-210}^{+200}$ & 38.4 & 1.8 & 0.01 & 0.14 & $301.9 / 245$ \\
\hline NGC 3147 & 2.9 & $0.07_{-0.01}^{+0.01}$ & $1.56_{-0.03}^{+0.04}$ & - & $144_{-55}^{+101}$ & 155.8 & 31.1 & - & - & $562.1 / 585$ \\
\hline NGC 3227 & 2.0 & $6.42_{-0.17}^{+0.15}$ & $1.44_{-0.04}^{+0.04}$ & - & $152_{-41}^{+22}$ & 866.3 & 43.9 & 0.06 & 10.12 & $2089.4 / 2082$ \\
\hline NGC 3254 & 1.8 & $<0.12$ & 1.9 (fixed) & - & - & 0.7 & 0.05 & - & - & $4.23 / 5$ \\
\hline NGC 3486 & 1.7 & $8.42_{-4.17}^{+28.9}$ & 1.9 (fixed) & $0.37_{-0.05}^{+0.06}$ & - & 8.5 & 0.06 & - & - & $30.29 / 44$ \\
\hline NGC $3516^{\star}$ & 3.5 & $1.04_{-0.49}^{+0.32}$ & $1.58_{-0.04}^{+0.02}$ & - & $180_{-16}^{+13}$ & 1460.3 & 264.1 & - & - & $2483.9 / 2056$ \\
\hline NGC 3735 & 1.3 & $15.23_{-10.17}^{+55.43}$ & $2.85_{-0.49}^{+0.67}$ & - & - & 17.21 & 3.4 & 0.11 & - & $139.7 / 131$ \\
\hline NGC 3941 & 1.9 & $<0.09$ & $2.02_{-0.24}^{+0.35}$ & - & - & 4.19 & 0.2 & & & $87.97 / 84$ \\
\hline NGC 3976 & 1.1 & $0.12_{-0.06}^{+0.07}$ & $2.01_{-0.03}^{+0.04}$ & - & - & 7.3 & 1.2 & - & - & $109.42 / 109$ \\
\hline NGC3982 & 1.0 & $43.23_{-16.96}^{+28.11}$ & $2.53_{-0.42}^{+0.44}$ & $0.28_{-0.04}^{+0.04}$ & $802_{-420}^{+678}$ & 16.60 & 0.6 & 0.025 & - & $109.42 / 109$ \\
\hline NGC $4051^{\star}$ & 1.2 & $0.53_{-0.35}^{+0.21}$ & $2.08_{-0.18}^{+0.11}$ & & $155_{-18}^{+14}$ & 650.32 & 22.4 & - & 9.7 & $2315.34 / 2225$ \\
\hline NGC 4138 & 1.3 & $8.85_{-0.44}^{+0.41}$ & $1.63_{-0.05}^{+0.05}$ & - & $80_{-34}^{+41}$ & 554.3 & 19.1 & 0.009 & - & $473.04 / 449$ \\
\hline NGC $4151^{\star}$ & 2.3 & $5.14_{-0.14}^{+0.13}$ & $1.55_{-0.03}^{+0.03}$ & - & $69_{-10}^{+11}$ & 21000.5 & 1034.4 & - & 2.54 & $109.42 / 109$ \\
\hline NGC 4168 & 2.4 & $<0.06$ & $2.02_{-0.12}^{+0.14}$ & - & - & 0.6 & 0.2 & - & - & $72.2 / 73$ \\
\hline NGC 4169 & 1.7 & $13.47_{-3.31}^{+6.07}$ & $2.01_{-0.44}^{+0.79}$ & - & & 23.5 & 6.9 & 0.017 & - & $22.87 / 30$ \\
\hline NGC4258* & 1.6 & $6.8_{-0.28}^{+0.35}$ & $1.62_{-0.05}^{+0.07}$ & - & $41_{-19}^{+17}$ & 380.9 & 2.1 & - & - & $1227.17 / 1258$ \\
\hline NGC4378 & 1.7 & $0.18_{-0.06}^{+0.06}$ & $1.55_{-0.15}^{+0.19}$ & - & & 14.7 & 2.1 & & & $31.84 / 38$ \\
\hline NGC4388 & 2.6 & $32.14_{-1.05}^{+1.19}$ & $1.86_{-0.08}^{+0.09}$ & - & $173_{-32}^{+15}$ & 2007.3 & 70.8 & 0.006 & & $1050.7 / 923$ \\
\hline NGC 4395^ & 1.9 & $1.02_{-0.10}^{+0.11}$ & $1.18_{-0.02}^{+0.03}$ & - & $80_{-9}^{+16}$ & 590.5 & 1.2 & - & - & $2298.85 / 2008$ \\
\hline NGC $4472^{\star}$ & 1.5 & $<0.82$ & $1.65_{-0.14}^{+0.26}$ & $0.87_{-0.11}^{+0.15}$ & - & 21.5 & 0.7 & - & - & $283.25 / 292$ \\
\hline NGC 4477 & 2.4 & $<0.02$ & $2.12_{-0.17}^{+0.25}$ & $0.41_{-0.16}^{+0.04}$ & - & 3.7 & 0.1 & - & - & $62.9 / 58$ \\
\hline NGC 4501 & 2.6 & $<0.06$ & $1.95_{-0.18}^{+0.19}$ & $0.66_{-0.01}^{+0.01}$ & - & 0.6 & 0.2 & - & - & $40.31 / 32$ \\
\hline NGC 4565 & 1.2 & $0.16_{-0.03}^{+0.03}$ & $1.87_{-0.09}^{+0.14}$ & & - & 20.7 & 0.2 & - & - & $102.5 / 88$ \\
\hline NGC 4639 & 2.2 & $<0.04$ & $1.79_{-0.05}^{+0.06}$ & - & - & 48.2 & 1.6 & - & - & $268.42 / 250$ \\
\hline NGC 4698 & 1.8 & $<0.07$ & $1.73_{-0.27}^{+0.22}$ & - & - & 4.8 & 0.1 & - & - & $33.82 / 33$ \\
\hline NGC 4725 & 0.8 & $<0.03$ & $2.68_{-0.23}^{+0.27}$ & $0.23_{-0.03}^{+0.03}$ & - & 2.3 & 0.04 & - & - & $64.4 / 71$ \\
\hline NGC5033 & 1.1 & $<0.04$ & $1.72_{-0.02}^{+0.02}$ & - & $286_{-71}^{+81}$ & - & 440.6 & 18.3 & - & $999.1 / 974$ \\
\hline NGC 5194* & 1.8 & $<0.65$ & $1.16_{-0.23}^{+0.14}$ & - & $1730_{-275}^{+422}$ & 25.5 & 0.2 & - & - & $89.33 / 91$ \\
\hline NGC $5273^{\star}$ & 0.9 & $0.72_{-0.09}^{+0.07}$ & $1.44_{-0.09}^{+0.07}$ & - & $191_{-72}^{+52}$ & 706.7 & 38.2 & - & - & $612.24 / 635$ \\
\hline NGC 6951 & 12.4 & $0.40_{-0.15}^{+0.31}$ & $2.59_{-0.30}^{+0.53}$ & $0.67_{-0.18}^{+0.16}$ & - & 4.8 & 0.3 & - & - & $32.33 / 55$ \\
\hline NGC 7479 & 5.3 & $40.2_{-8.75}^{+11.44}$ & $2.56_{-0.16}^{+0.15}$ & $0.27_{-0.07}^{+0.07}$ & $480_{-390}^{+210}$ & 22.5 & 2.8 & 0.004 & - & $137.3 / 133$ \\
\hline NGC 7743 & 4.8 & $0.3_{-0.14}^{+0.18}$ & $3.63_{-1.03}^{+1.14}$ & $0.23_{-0.06}^{+0.06}$ & - & 1.0 & 0.07 & - & - & $16.7 / 18$ \\
\hline
\end{tabular}

Column 1: Galaxy name. Column 2: galactic column density in units of $10^{20} \mathrm{~cm}^{-2}$. Column 3: observed column density in units of $10^{22} \mathrm{~cm}^{-2}$. Column 4: power-law photon index of the continuum emission. Column 5: temperature of the Raymond-Smith model. Column 6: equivalent width of the $\mathrm{FeK}_{\alpha}$ emission. Column 7: observed 2-10 keV flux in units of $10^{-14} \mathrm{erg} \mathrm{s}^{-1} \mathrm{~cm}^{-2}$. Column 8: observed 2-10 keV luminosity in units of $10^{40} \mathrm{erg} \mathrm{s}^{-1}$. Column 9: ratio of the normalizations of the scattered to the continuum emission. Column 10: ratio of the normalizations of the reflected to the continuum emission. Column 11: reduced $\chi^{2} .{ }^{\star}$ Indicates source with very complex spectra for which only a rough parametrization in the $2-10 \mathrm{keV}$ band is presented here.

comes from large (usually kpc) scales, remains unabsorbed while the X-ray flux is diminished because of absorption.

These criteria however should be considered with caution. For example high Equivalent Width (EW) lines may also appear in the case of anisotropic distribution of the scattering medium (Ghisellini et al. 1991), or in the case where there is a time lag between the reprocessed and the direct component (e.g. NGC 2992, Weaver et al. 1996). Also there have been reports of Compton-thick sources where the value of $\mathrm{FeK}_{\alpha}$ line $E W$ is well below $1 \mathrm{keV}$ (e.g. Awaki et al. 2000, for Mkn1210).

In Fig. 1 we plot the column density obtained from the spectral fittings as a function of the X-ray to optical flux ratio, 


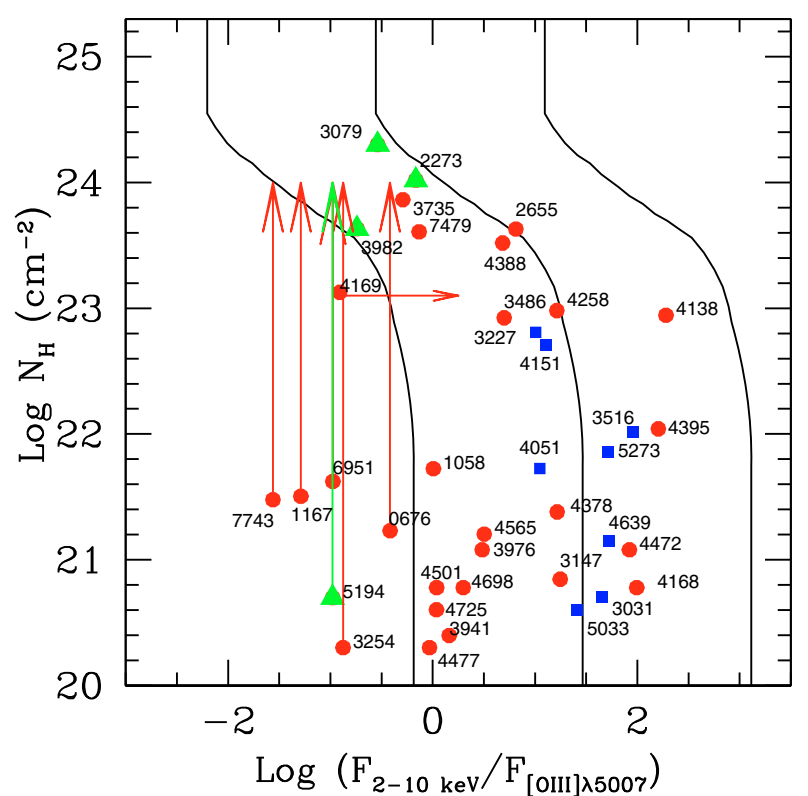

Fig. 1. Distribution of the $N_{\mathrm{H}}$ values as a function of the $F_{2-10 \mathrm{keV}} / F_{[\mathrm{OIII}]}$ ratio. Filled boxes and circles denote Seyfert- 1 and Seyfert-2 galaxies respectively. The triangles show Seyfert-2 galaxies with large $E W(>700 \mathrm{eV})$. The solid lines represent the mean $N_{\mathrm{H}}$ vs. $F_{2}-10 \mathrm{keV} / F_{[\mathrm{OIII}]}$ relation followed by the Seyfert- 1 in our sample together with the $\pm 3 \sigma$ dispersion (see text).

$F_{2-10 \mathrm{keV}} / F_{\text {[OIII] }}$. The [OIII] fluxes are corrected for the optical reddening using the formula described in Basanni et al. (1999): $F_{\text {[OIII }]_{\mathrm{COR}}}=F_{[\mathrm{OIII}]_{\mathrm{OBS}}}\left[\left(\mathrm{H}_{\alpha} / \mathrm{H}_{\beta}\right) /\left(\mathrm{H}_{\alpha} / \mathrm{H}_{\beta}\right)_{\mathrm{o}}\right]^{2.94}$, where the intrinsic Balmer decrement $\left(\mathrm{H}_{\alpha} / \mathrm{H}_{\beta}\right)_{\mathrm{o}}$ equals 3 .

The solid lines in Fig. 1 show the expected correlation between these quantities, assuming a photon index of 1.8 and $1 \%$ reflected radiation (see also Maiolino et al. 1998; Cappi et al. 2006). The starting point in the $x$-axis for the middle solid line is determined by averaging the $F_{2-10 \mathrm{keV}} / F_{\text {[OIII] }}$ values of the Seyfert-1 population only, while the lines at right and left show the $3 \sigma$ dispersion. The sources occupying the low $\left(F_{2-10 \mathrm{keV}} / F_{[\mathrm{OIII}]}, N_{\mathrm{H}}\right)$ region in this plot could be possibly highly obscured or Compton-thick AGN.

In two cases (NGC 2273, NGC 3079) we can immediately tell the presence of a Compton-thick nucleus through the presence of an absorption turnover in the spectral fittings. Both sources present high values of the $\mathrm{FeK}_{\alpha}$ line $E W(>700 \mathrm{eV})$. One more source (NGC 5194), despite the fact that it presents the highest value of $\mathrm{FeK}_{\alpha}(\sim 1700 \mathrm{eV})$, shows no absorption at all. However, the very flat $\mathrm{X}$-ray spectrum and the very low $F_{2-10 \mathrm{keV}} / F_{\text {[OIII] }}$ value further suggest that this is a highly obscured or a Compton-thick source. According to the $N_{\mathrm{H}}-F_{2-10 \mathrm{keV}} / F_{\text {[OIII] }}$ relation a minimum value for the $N_{\mathrm{H}}$ is $4 \times 10^{23} \mathrm{~cm}^{-2}$ (see Fig. 1).

There are also 5 Seyfert-2 galaxies (NGC 676, NGC 1167, NGC 3254, NGC 6951 and NGC 7743) occupying the low $F_{2-10 \mathrm{keV}} / F_{[\mathrm{OIII}]}$ regime. We do not consider NGC 4169 because of the large error in the estimation of the [OIII] flux (see Ho et al. 1997). These, according to the expected $N_{\mathrm{H}}-F_{2-10 \mathrm{keV}} / F_{\text {[OIII] }]}$ relation, should present high values of $N_{\mathrm{H}}$. According to Fig. 1, the minimum $N_{\mathrm{H}}$ value is $\sim 2 \times 10^{23} \mathrm{~cm}^{-2}$ for NGC $676, \sim 6 \times$ $10^{23} \mathrm{~cm}^{-2}$ for NGC 6951 and $\sim 10^{24} \mathrm{~cm}^{-2}$ for NGC 1167 and NGC 7743. However, the X-ray spectral fittings show low absorption $\left(<10^{22} \mathrm{~cm}^{-2}\right)$ while in addition there is no indication for a flat photon index or strong $\mathrm{FeK}_{\alpha}$ line. This may be due to the limited photon statistics in the hard $(>2 \mathrm{keV})$ band, which

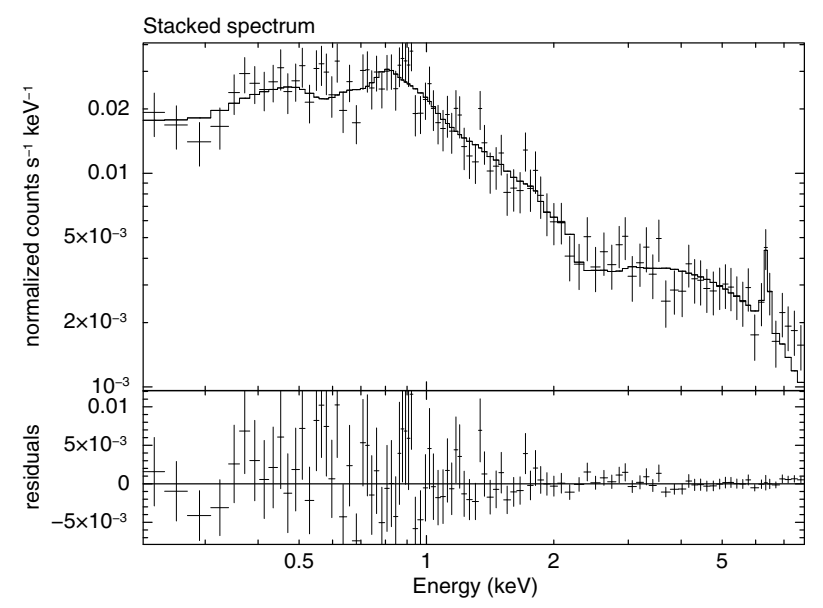

Fig. 2. Stacked X-ray spectrum of the Seyfert-2 Galaxies NGC 0676, NGC 1167, NGC 3254, NGC 6951 and NGC 7743. The best fit model and residuals are also shown.

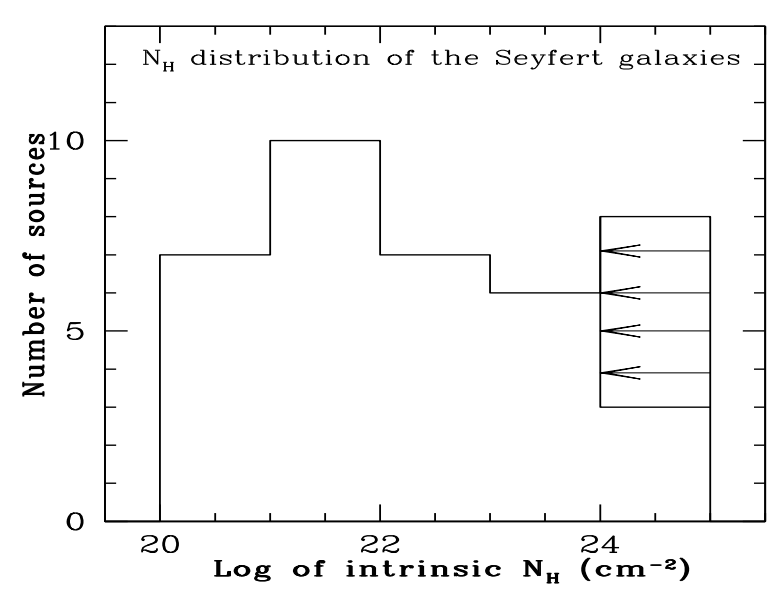

Fig. 3. The estimated $N_{\mathrm{H}}$ distribution of the Seyfert galaxies in our sample (solid line). The vertical arrows in the last $N_{\mathrm{H}}$ bin $\left(\log \left(N_{\mathrm{H}}\right)>\right.$ $24 \mathrm{~cm}^{-2}$ ) show the upper and the lower limits for the number of the the Compton-thick sources in our sample.

does not allow us to examine in detail the spectral characteristics. Note however, that in the spectrum of at least two sources (NGC 1167 and NGC 7743) there is some indication for a flattening at hard energies which could suggest a heavily buried or reflected component. We investigate further this issue by deriving the mean, stacked X-ray spectrum. We use the MATHPHA tasks of FTOOLS to derive the weighted stacked X-ray spectrum of the five EPIC-PN observations. The corresponding ancillary files are also produced using ADDRMF and ADDARF tasks of FTOOLS. We perform no correction for the rest-frame energy because the differences in the redshifts are negligible. An absorbed power-law model plus a Gaussian line and a soft excess component (Raymond-Smith model) reproduce well the mean spectrum (Table 4). In Fig. 2 we present the data along with the best-fit. The average spectrum shows significant absorption consistent with the measured value of the $\mathrm{FeK}_{\alpha}$ line EW.

Our results above can be summarised as follows. The number of absorbed nuclei $\left(N_{\mathrm{H}}>10^{22} \mathrm{~cm}^{-2}\right)$ are 21 out of 38 or $55 \pm 12 \%$. The number of Compton-thick sources is three i.e. $8 \pm 5 \%$ although, if we adopt the extreme case where all the low $F_{2-10 \mathrm{keV}} / F_{\text {[OIII] }}$ host Compton-thick nuclei this number would rise to 8 or $21 \pm 7 \%$. Our estimates on the amount of $N_{\mathrm{H}}$ in the local universe are illustrated in Fig. 3. The solid line describes 
Table 4. The stacked X-ray spectrum of the 5 Seyfert-2 galaxies with low $F_{2-10 \mathrm{keV}} / F_{[\mathrm{OIII}]}$.

\begin{tabular}{ccccc}
\hline \hline$N_{\mathrm{H}}\left(\mathrm{cm}^{-2}\right)$ & $\Gamma$ & $k T(\mathrm{keV})$ & $E W_{\mathrm{FeK}}(\mathrm{eV})$ & $\chi_{v}^{2}$ \\
\hline $10.30_{-1.91}^{+2.01}$ & $1.71_{-0.17}^{+0.08}$ & $0.73_{-0.12}^{+0.13}$ & $255_{-146}^{+243}$ & $72.41 / 91$ \\
\hline
\end{tabular}

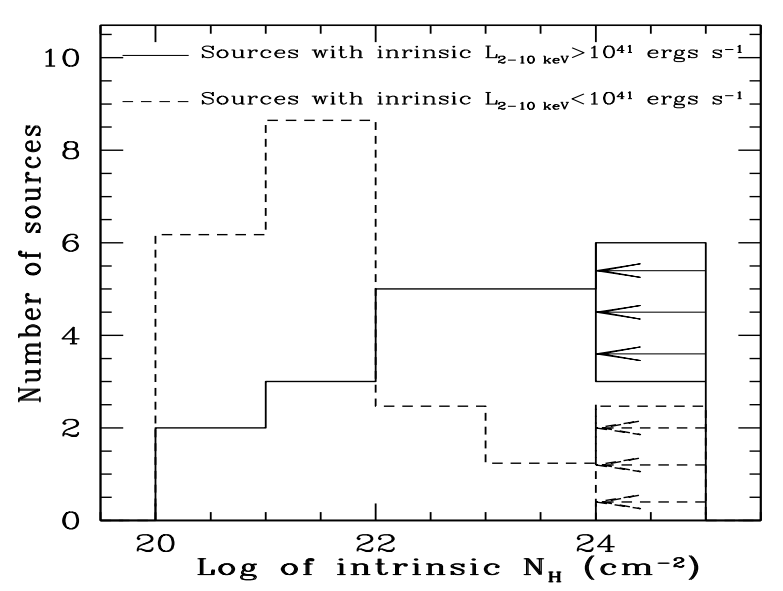

Fig. 4. The derived $N_{\mathrm{H}}$ distribution of the Seyfert galaxies in the bright (solid line) and the faint (dashed line) sub-samples. The vertical arrows in the Compton-thick regime $\left(\log \left(N_{\mathrm{H}}\right)>24 \mathrm{~cm}^{-2}\right)$ define the upper and lower limit for the number of the Compton-thick sources in our sample.

the $N_{\mathrm{H}}$ distribution. The vertical arrows in the highest $N_{\mathrm{H}}$ bin show the upper and lower limits for the number of Comptonthick sources.

\subsection{The absorption in the bright sub-sample}

Our findings should play an important role to the XRB synthesis models (Comastri et al. 1995; Gilli et al. 2007). Gilli et al. (2007) assume in their models a lower luminosity of $10^{41} \mathrm{erg} \mathrm{s}^{-1}$. However, the intrinsic 2-10 keV luminosity of our sources starts from as low as a few times $10^{38} \mathrm{erg} \mathrm{s}^{-1}$ which is about 3 orders of magnitude lower. Therefore it is useful to present our results separately for the fainter $\left(L_{2-10 \mathrm{keV}}<10^{41} \mathrm{erg} \mathrm{s}^{-1}\right)$ and the brighter $\left(L_{2-10 \mathrm{keV}}>10^{41} \mathrm{erg} \mathrm{s}^{-1}\right)$ sub-sample containing 21 and 17 sources respectively (see Fig. 4 ). The intrinsic $L_{X}$ values are determined using the best fitting results. For the three Comptonthick sources the intrinsic $L_{X}$ has been estimated assuming that $1 \%$ of the intrinsic luminosity is actually observed below $10 \mathrm{keV}$ due to scattering and or reflection (e.g. Comastri 2004). In the bright sample the fraction of the highly absorbed sources is $\sim 75 \%$ and the Compton-thick sources most probably account for $15-20 \%$ of the total population. This fraction can reach a maximum of $29 \%$ in the unlikely case where all the low $F_{2}-$ $10 \mathrm{keV} / F_{[\mathrm{OIII}]}$ sources host a Compton-thick nucleus.

\subsection{Unabsorbed Seyfert-2 galaxies}

The X-ray spectral analysis reveals several Seyfert-2 galaxies with very little or no X-ray absorption. As we have already discussed some of these, i.e. the five with low X-ray to [OIII] flux ratio are most probably associated with a highly obscured or even a Compton-thick nucleus. In Fig. 1 there are 12 additional Seyfert-2 galaxies (NGC 1058, NGC 3147, NGCC 3941, NGC 3976, NGC 4168, NGC 4378, NGC 4472, NGC 4477, NGC 4501,NGC 4565,NGC 4698 and NGC 4725) with $N_{\mathrm{H}}$ less than $10^{22} \mathrm{~cm}^{-2}$ but an average $F_{2-10 \mathrm{keV}} / F_{\text {[OIII] }}$ value. This behaviour is not unknown (e.g. Pappa et al. 2001;
Gliozzi et al. 2007). In particular NGC 3147 is a well established example, through simultaneous optical and X-ray observations, of a spectroscopically classified Seyfert- 2 galaxy with very little or no absorption (Bianchi et al. 2008). NGC 4698 and NGC 4565 have also been discussed to be good candidates, (see Georgantopoulos \& Zezas 2003; Panessa \& Bassani 2002).

It is possible that some of our new unabsorbed candidates are contaminated by nearby luminous X-ray sources that we are unable to resolve owing to the X-ray telescope's angular resolution. An inspection of the available Chandra images which have a superior resolution ( 0.5 arcsec FWHM) could be very helpful towards this direction. All but three sources (NGC 3941, NGC 3976 and NGC 4378) present archival Chandra data. Although a detailed analysis of the properties of the unabsorbed Seyfert-2 galaxies is the scope of a forthcoming paper, we briefly report on whether there is any evidence for contamination. NGC 1058 and NGC 4168 are significantly contaminated from nearby luminous X-ray sources (see also Foschini et al. 2002; Cappi et al. 2006) while NGC 4472 suffers from very strong diffuse emission. Finally, inspection of XMM-Newton images show that NGC 3941 and NGC 4501 are contaminated (less than $30 \%$ of the counts) by nearby sources (see also Foschini et al. 2002; Cappi et al. 2006).

We further try to examine the X-ray properties of unobscured Seyfert- 2 galaxies by deriving their stacked spectrum. We use MATHPHA task of FTOOLS software to create the weighted mean X-ray spectrum of the EPIC-PN observations. Weighted mean ancillary files are produced using the ADDRMF and ADDARF tasks of FTOOLS. NGC 3147, NGC 4565 and NGC 4698 are not considered in the mean spectrum since there is already evidence that they do not present any absorption. We also exclude the five contaminated sources leaving the cases of NGC 3976, NGC 4725, NGC 4378 and NGC 4477 to be considered.

We try to detect any spectral feature, such as the $\mathrm{FeK}_{\alpha}$ line, that could give away the presence of a hidden nucleus in this population as marginally suggested in some cases (e.g. Brightman \& Nandra 2008). We fit the average spectrum with an absorbed power-law model plus a Raymond-Smith model. The spectral fitting results are listed in table 5. There is no significant evidence for the presence of an $\mathrm{FeK}_{\alpha}$ emission line. Nevertheless, if we choose to include a Gaussian component, the upper limit of the $E W$ is $\sim 600 \mathrm{eV}$ at the 90 per cent confidence level. In Fig. 6 we present the mean spectrum along with the best fit model and the residuals. Assuming that all these sources are truly unabsorbed Seyfert-2 galaxies then their total fraction accounts for $\sim 20$ per cent of the total population.

It has been proposed that the unabsorbed Seyfert-2 galaxies are "naked" nuclei i.e. they lack a Broad-Line-Region, BLR, (see Ho 2008, for a review). Various theoretical models could explain this behaviour. Nicastro (2000) presented a model which relates the width of the Broad Emission Lines of AGN to the Keplerian velocity of an accretion disk at a critical distance from the central black hole. Under this scheme the Broad Line Region is linked to the accretion rate of the AGN i.e. below a minimum accretion rate the BLR cannot form. Recently Elitzur \& Shlosman (2006) presented an alternative model which depicts the torus as 
Table 5. The stacked X-ray spectrum of the unabsorbed Seyfert-2 galaxies.

\begin{tabular}{ccccc}
\hline \hline$N_{\mathrm{H}}\left(\mathrm{cm}^{-2}\right)$ & $\Gamma$ & $k T(\mathrm{keV})$ & $E W_{\mathrm{FeK}}(\mathrm{eV})$ & $\chi_{v}^{2}$ \\
\hline$<0.1$ & $2.02_{-0.15}^{+0.18}$ & $0.37_{-0.08}^{+0.07}$ & $<600$ & $118.6 / 125$ \\
\hline
\end{tabular}

Table 6. Accretion rates and luminosities for the unabsorbed Seyfert-2 galaxies.

\begin{tabular}{cccc}
\hline \hline Name & $\log \left(M_{B H} / M_{\odot}\right)$ & $\log \left(L_{\mathrm{BOL}}\right)$ & $\begin{array}{c}L_{\mathrm{BOL}} / L_{\mathrm{EDD}} \\
\times 10^{-4}\end{array}$ \\
\hline NGC 1058 & 4.9 & 39.8 & 5.5 \\
NGC 3147 & 8.8 & 43.0 & 1.1 \\
NGC 4168 & 7.9 & 41.3 & 0.15 \\
NGC 4378 & 7.9 & 41.8 & 0.50 \\
NGC 4472 & 8.8 & 41.4 & 0.026 \\
NGC 4477 & 7.9 & 40.5 & 0.025 \\
NGC 4501 & 7.9 & 41.3 & 0.17 \\
NGC 4725 & 7.5 & 40.1 & 0.027 \\
NGC 4565 & 7.7 & 40.8 & 0.084 \\
NGC 4698 & 7.8 & 40.5 & 0.030 \\
\hline
\end{tabular}

Column 1: name. Column 2: black hole mass in units of Solar Masses. Column 3: bolometric luminosity in units of erg s ${ }^{-1}$. Column 4: accretion rate $L_{\mathrm{BOL}} / L_{\mathrm{EDD}}$.

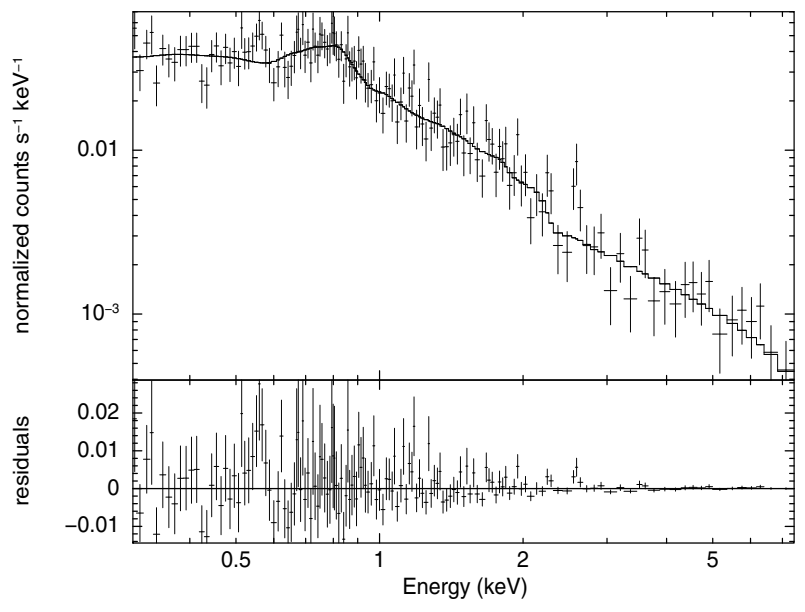

Fig. 6. Stacked X-ray spectrum of the unabsorbed Seyfert-2 Galaxies. The best fit model and the residuals are also shown.

the inner region of clumpy wind outflowing from the accretion disc. According to this model the torus and the BLR disappear when the bolometric luminosity decreases below $\sim 10^{42} \mathrm{erg} \mathrm{s}^{-1}$ because the accretion onto the central black hole can no longer sustain the required cloud outflow rate.

In Table 6 we try to compare our results with the above model predictions. In Col. 1 we give an estimate for the mass of the central Black Hole, taken from Panessa et al. (2006) and McElroy (1995). The mass estimation is inferred from the massvelocity dispersion correlation. In the case of NGC 3976 there is no information available in the literature. In Col. 2 we calculate the bolometric luminosities using the corrections determined by Elvis et al. (1994) i.e., $L_{\mathrm{BOL}}=35 \times L_{2-10 \mathrm{keV}} \mathrm{erg} \mathrm{s}^{-1}$. In Col. 3 we give the accretion rate estimator given by $L_{\mathrm{BOL}} / L_{\mathrm{EDD}} \simeq 1.3 \times 10^{38} M / M_{\odot}$. The Eddington Luminosity $\left(L_{\mathrm{EDD}}\right)$ is given by $L_{\mathrm{EDD}}=4 \pi G M m_{\mathrm{p}} c / \sigma_{\mathrm{T}}$ where $M$ is the black hole mass, $m_{\mathrm{p}}$ is the proton mass, $\sigma_{\mathrm{T}}$ is the Thomson scattering cross section.

All the sources present very low accretion rates, well below the threshold of $1-4 \times 10^{-3}$ proposed by Nicastro (2000) and Nicastro et al. (2003). Furthermore all these sources (but NGC 3147) present very low bolometric luminosities also below the critical value of $10^{42} \mathrm{erg} \mathrm{s}^{-1}$ predicted by Elitzur \& Shlosman (2006). This supports the idea that the key parameter is not the orientation but an intrinsic parameter (low accretion rate or luminosity), which prevents the formation of the BLR.

\section{Discussion}

\subsection{Comparison with other optically selected samples}

In this work we present XMM-Newton observations of all the Seyfert galaxies from the Palomar survey (Ho et al. 1995). We find that $\sim 50$ per cent of the Seyfert population is absorbed by $N_{\mathrm{H}}>10^{22} \mathrm{~cm}^{-2}$. In this sample we have identified 3 Comptonthick sources which translates to a fraction of $\sim 8$ per cent. Five more sources possibly host a highly absorbed or a Compton thick nucleus. In the very extreme, and rather unlikely case were all these candidates are true Compton-thick sources their fraction reaches 20 per cent of the total population.

Cappi et al. (2006) and Pannesa et al. (2006), also using data from the Palomar survey, provide estimates for the fraction of obscured AGN in the local universe. These authors find that about $50 \%$ of their sources are obscured $\left(>10^{22} \mathrm{~cm}^{-2}\right)$. Their estimates on the fraction of Compton thick sources suggest an absolute minimum of 20 per cent of the total population. This result comes in contradiction with our findings. However their sample includes 2 objects not fulfilling the Palomar Survey selection criteria (see also Sect. 2). These are the 2 Compton-thick AGN NGC 1068 and NGC 3185. When we exclude these an agreement is found.

Risaliti et al. (1999) study the X-ray absorption in a sample of 45 Seyfert-2 galaxies finding that a considerable fraction of these are associated with Compton-thick nuclei. A direct comparison with our results is not straightforward since these authors exclude all the sources with $F_{[\mathrm{OIII}]}>4 \times 10^{-13} \mathrm{erg} \mathrm{cm}^{-2} \mathrm{~s}^{-1}$. However we think that only a luminosity cutoff could reveal column density distribution of the population that contributes to the XRB (see Sect. 5.2).

\subsection{X-ray background synthesis models}

The XRB synthesis models can provide tight constraints on the number density of Compton-thick sources. These models attempt to fit the spectrum of the X-ray background roughly in 

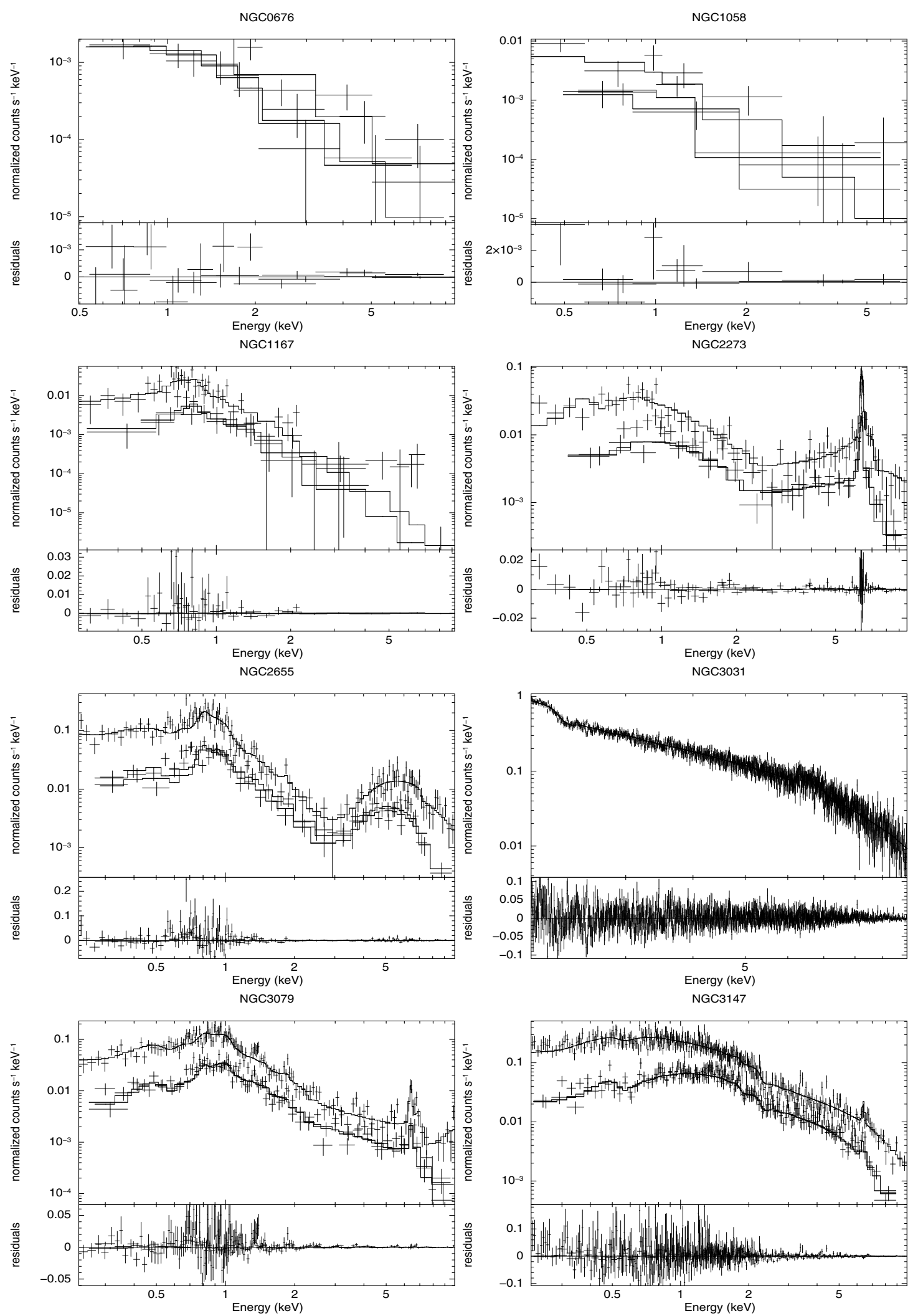

Fig. 6. The XMM-Newton X-ray spectra for all the sources in our sample. The upper panel shows the X-ray spectrum and the best fit model listed in Table 3 and the lower panel the residuals.

the $1-100 \mathrm{keV}$ range. It is well established that a large number of Compton-thick sources is needed (Gilli et al. 2007; but see Treister et al. 2009) to reproduce the hump of the X-ray background spectrum at $30-40 \mathrm{keV}$ where most of its energy density lies (Churazov et al. 2007; Frontera et al. 2007). Here, we compare the fraction of the Compton-thick sources predicted by the model of Gilli et al. (2007) with our results. We use the publicly 

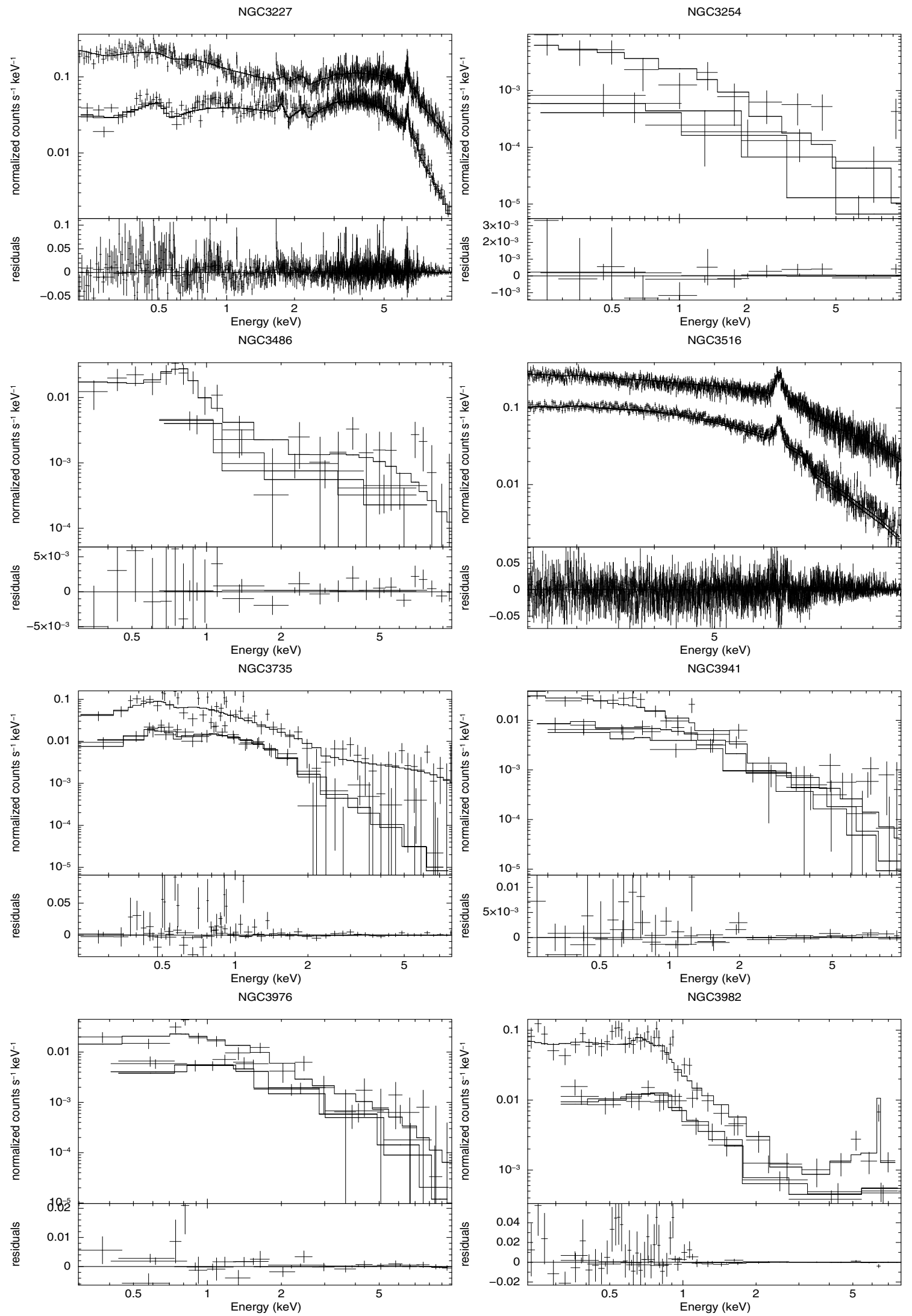

Fig. 6. continued.

available POMPA software ${ }^{1}$. This predicts the number counts at a given redshift, flux and luminosity range using the best-fit results for the fraction of obscured, Compton-thick sources, of the X-ray background synthesis model of Gilli et al. (2007). 

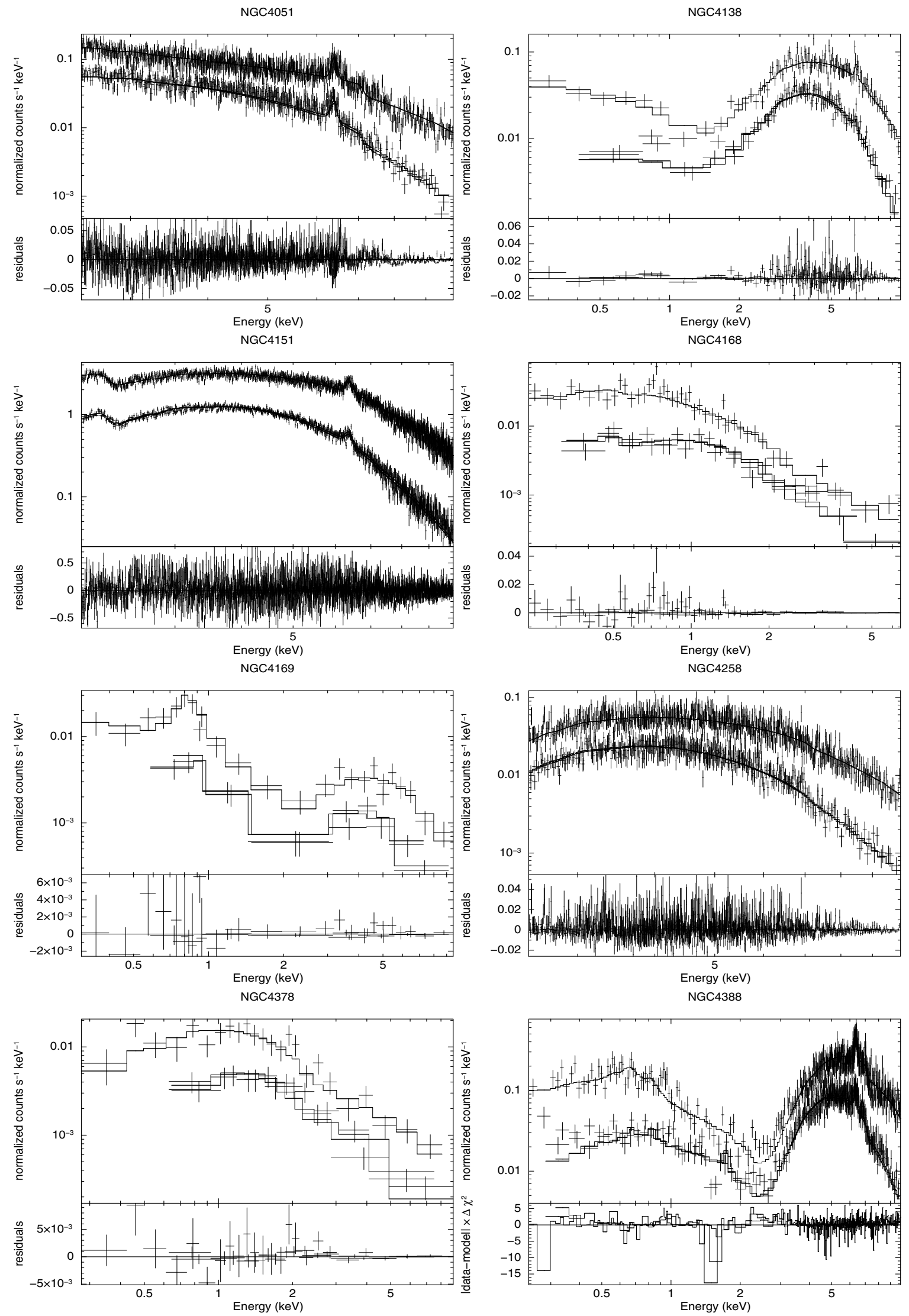

Fig. 6. continued.

We restrict the comparison to low redshifts $z<0.017$ and $\mathrm{X}$-ray luminosities in the bin $10^{41}<L_{2}-10 \mathrm{keV}<10^{44}$. As our sample is not flux limited in the X-rays, we have to choose a flux limit deep enough to ensure that all sources in this luminosity 

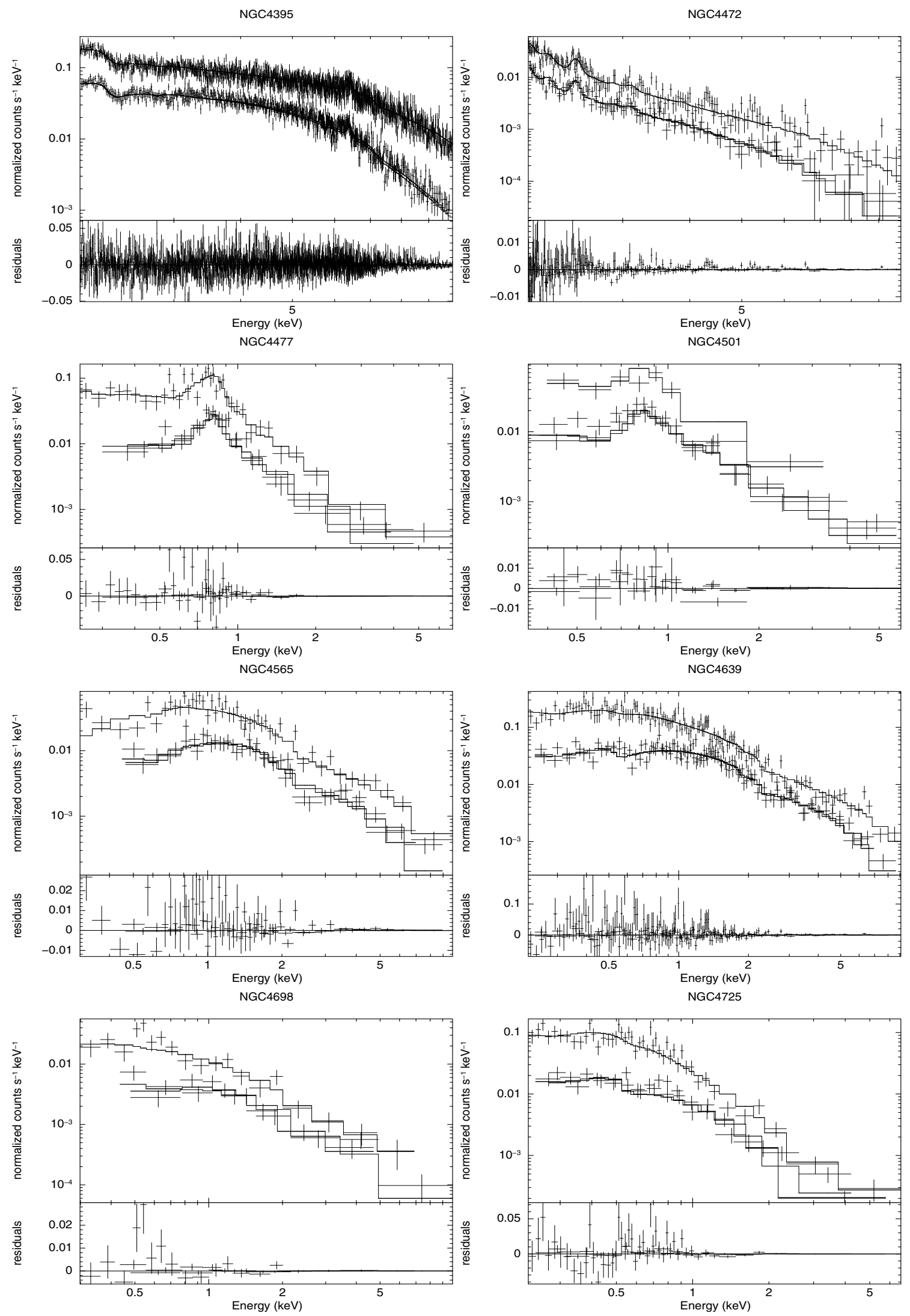

Fig. 6. continued.

and redshift bin are detected. A flux limit of $10^{-14} \mathrm{erg} \mathrm{cm}^{-2} \mathrm{~s}^{-1}$ satisfies this constraint. The X-ray background synthesis models predict a fraction of Compton-thick sources of about $40 \%$ which is higher compared with our results $15 \pm 8 \%$. Only if all the low $F_{X} / F_{\text {[OIII] }}$ Seyfert-2 galaxies are associated with a 

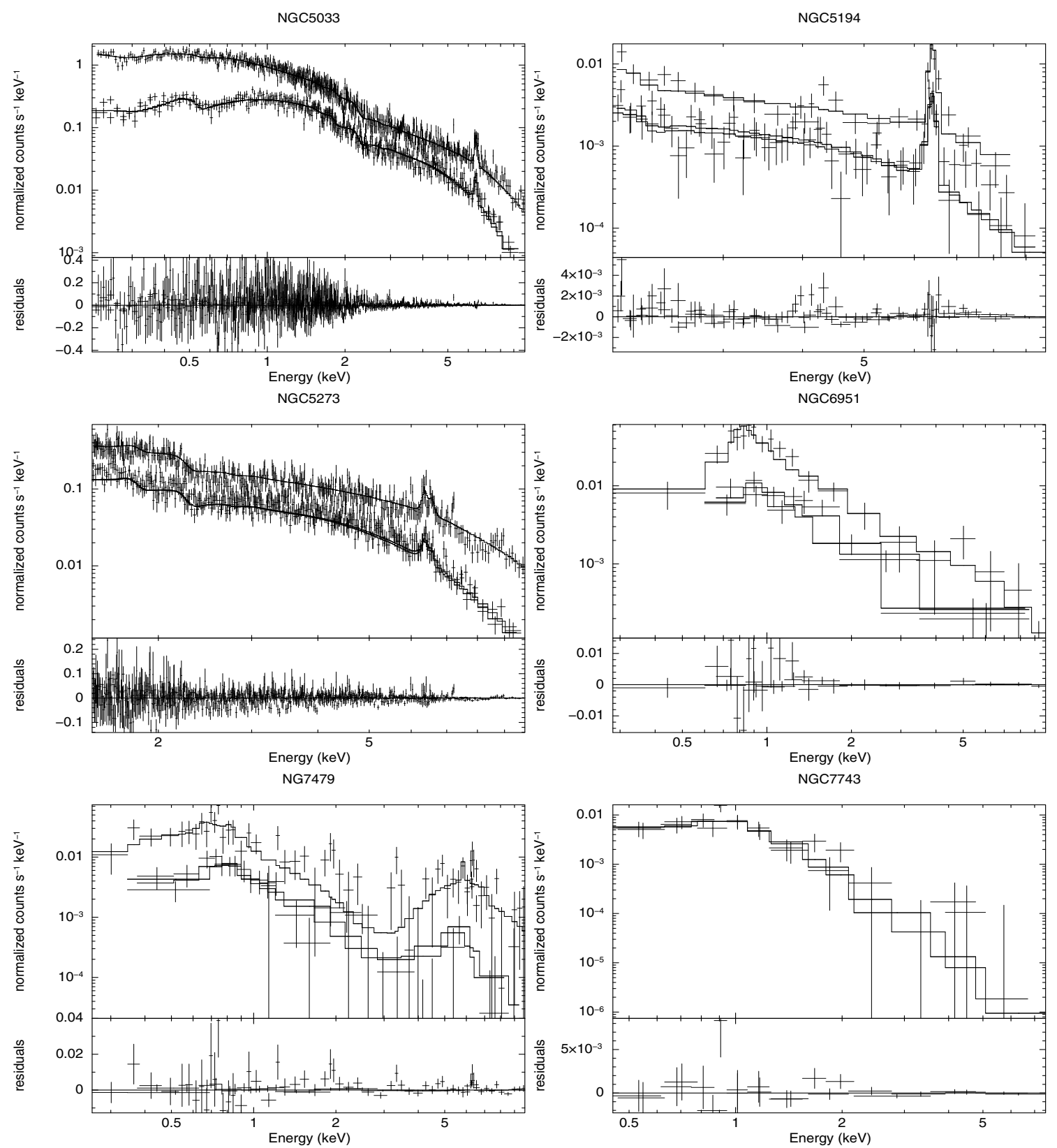

Fig. 6. continued.

Compton-thick nuclei the discrepancy would become less pronounced.

Optically selected samples can still miss a fraction of Compton-thick AGN. For example, NGC 6240 is classified as a LINER in the optical while BeppoSAX observations show the presence of a Compton-thick nucleus (Vignati et al. 1999). Moreover SUZAKU observations (Ueda et al. 2007; Comastri et al. 2007) have demonstrated that a small fraction of AGN may have a $4 \pi$ coverage, instead of the usually assumed toroidal structure. These sources will not exhibit the usual high excitation narrow emission lines and therefore will not be classified as AGN on the basis of their optical spectrum.

Recent results based on INTEGRAL and SWIFT observations reveal a small fraction of Compton-thick sources (e.g. Treister et al. 2009; Sazonov et al. 2008; Sazonov et al. 2007; Ajello et al. 2008). In particular at the flux limit of $\sim 10^{-11} \mathrm{erg} \mathrm{cm}^{-2} \mathrm{~s}^{-1}$ in the
17-60 keV energy band, INTEGRAL observations find 10-15\% Compton-thick sources. The SWIFT/BAT hard X-ray survey failed to identify any Compton-thick AGN. This non detection discards the hypothesis that their fraction accounts for the 20 per cent of the total AGN at $>2 \sigma$ confidence level. It is true however that some heavily obscured Compton-thick sources with $N_{\mathrm{H}} \sim 10^{25-26} \mathrm{~cm}^{-2}$ would be missed even by these ultra hard X-ray surveys.

\subsection{Less absorption at very low luminosities}

In the low-luminosity sub-sample (intrinsic $L_{2}-10 \mathrm{keV}<$ $10^{41} \mathrm{erg} \mathrm{s}^{-1}$ ) the fraction of obscured sources diminishes to $30 \%$. This result comes in apparent contradiction with recent findings suggesting an increasing fraction of obscuration with decreasing luminosity (e.g. Akylas et al. 2006; La Franca et al. 2005). 
This behaviour may reflect a physical dependence of the column density with intrinsic luminosity as suggested by Elitzur \& Shlosman (2006). These authors present a model where the torus and the BLR disappear when the bolometric luminosity decreases below $\sim 10^{42}$ erg s$^{-1}$ because the accretion onto the central black hole can no longer sustain the required cloud outflow rate. It is interesting to note that the corresponding luminosity in the $2-10 \mathrm{keV}$ band is about several $\times 10^{40} \mathrm{erg} \mathrm{s}^{-1}$, assuming the Spectral Energy Distribution of Elvis et al. (1994). Interestingly, almost all of our Seyfert-2 sources with no absorption present luminosities below this limit (with the exception of NGC 3147). We note however, there are sources (NGC 3486, NGC 3982) with low luminosity, which present column densities around $10^{22}-10^{23} \mathrm{~cm}^{-2}$. Alternatively, it is possible that at least in a few cases, the large XMM-Newton Point Spread Function results in contamination by nearby sources. Thus the nuclear X-ray emission could be out-shined giving the impression that there is no obscuration (e.g. Brightman \& Nandra 2008). However, both the inspection of the Chandra images as well as the stacked spectrum of the unabsorbed sources do not favour such a scenario.

\section{Conclusions}

XMM-Newton observations are available for all 38 Seyfert galaxies from the Palomar spectroscopic sample of galaxies of Ho et al. $(1995,1997)$. Our goal is to determine the distribution of the X-ray absorption in the local Universe through X-ray spectroscopy. Our sample consists of 30 Seyfert-2 and 8 Seyfert- 1 galaxies. The results can be summarised as follows:

- We find a high fraction of obscured sources $\left(>10^{22} \mathrm{~cm}^{-2}\right)$ of about $50 \%$.

- A number of sources present low $F_{\mathrm{X}} / F_{\text {[OIII] }}$ ratio. Their individual spectra show no evidence of high absorbing column densities. However, their stacked spectrum shows significant amount of absorption $\left(\sim 3 \times 10^{23} \mathrm{~cm}^{-2}\right)$.

- Considering only the bright sub-sample $\left(L_{2-10 \mathrm{keV}}>\right.$ $10^{41} \mathrm{erg} \mathrm{s}^{-1}$ ), i.e. only these sources which contribute a significant amount to the X-ray background flux, we find that $75 \%$ of our sources are obscured.

- In the bright sub-sample there are at least 3 Compton-thick AGN translating to a fraction of $15 \%$ which is lower than the predictions of the X-ray background synthesis models at this luminosity and redshift range. Only if we consider, the rather unlikely scenario, where all Seyfert- 2 galaxies with a low $F_{\mathrm{X}} / F_{\text {[OIII] }}$ ratio are associated with Compton-thick sources we would alleviate this discrepancy.

- We find a large number of unobscured Seyfert-2 galaxies. All these have low luminosities $L_{2-10 \mathrm{keV}}<3 \times 10^{41} \mathrm{erg} \mathrm{s}^{-1}$. Inspection of the Chandra images, where available, demonstrates that in most cases these are not contaminated by nearby sources. Furthermore, their stacked spectrum reveals no absorption. It is most likely that these are genuinely unobscured sources in accordance with the predictions of the models of Elitzur \& Sloshman (2006).

\section{References}

Ajello, M., Rau, A., Greiner, J., et al. 2008, ApJ, 673, 96

Akylas, A., Georgantopoulos, I., Georgakakis, A., Kitsionas, S., \& Hatziminaoglou E. 2006, A\&A, 459, 693

Alexander, D. M., Bauer, F. E., Brandt, W. N., et al. 2003, AN, 324, 8 Awaki, H., Ueno, S., \& Taniguchi, Y. 2000, AdSpR, 25, 797

Barger, A. J., Cowie, L. L., Capak, D. M., et al. 2003, AJ, 126, 632

Barthelmy, S. D., Barbier, L. M., Cummings, J. R., et al. 2005, SSRv, 120, 143 Baskin, A., \& Laor, A. 2008, ApJ, 682, 110

Bassani, L., Dadina, M., Maiolino, R., et al. 1999, ApJS, 121, 473

Bassani, L., Molina, M., Malizia, A., et al. 2006, ApJ, 636, L65

Bianchi, S., Corral, A., Panessa, F., et al. 2008, MNRAS, 385, 195

Brandt, W. N., \& Hasinger, G. 2005, ARA\&A, 43, 827

Brightman, M., \& Nandra, K. 2008, MNRAS, 390, 1241

Cappi, M., Panessa, F., Bassani, L., et al. 2006, A\&A, 446, 459

Churazov, E., Sunyaev, R., Revnivtsev, M., et al. 2007, A\&A, 467, 529

Comastri, A. 2004, ASSL, 308, 245

Comastri, A., Setti, G., Zamorani, G., \& Hasinger, G. 1995, A\&A, 296, 1

Dickey, J. M., \& Lockman, F. J. 1990, ARA\&A, 28, 215

Elitzur, M., \& Shlosman, I. 2006, ApJ, 648, L101

Elvis, M., Wilkes, B. J., McDowell, J. C., et al. 1994, ApJS, 95, 1

Foschini, L., Di Cocco, G., Ho, L. C., et al. 2002, A\&A, 392, 817

Frontera, F., Orlandini, M., Landi, R., et al. 2007, ApJ, 666, 86

Georgantopoulos, I., \& Zezas, A. 2003, ApJ, 594, 704

Georgantopoulos, I., Georgakakis, A., \& Akylas, A. 2007, A\&A, 466, 823

Ghisellini, G., George, I. M., Fabian, A. C., \& Done, C. 1991, MNRAS, 248, 14 Gilli, R., Comastri, A., \& Hasinger, G. 2007, A\&A, 463, 79

Gliozzi, M., Sambruna, R. M., \& Foschini, L. 2007, ApJ, 662, 878

Guainazzi, M., Matt, G., \& Perola, G. C. 2005, A\&A, 444, 119

Heckman, T. M., Ptak, A., Hornschemeier, A., \& Kauffmann, G. 2005, ApJ, 634, 161

Ho, L. C. 2008, ARA\&A, 46, 475

Ho, L. C., \& Ulvestad, J. S. 2001, ApJS, 133, 77

Ho, L. C., Filippenko, A. V., \& Sargent, W. L. 1995, ApJS, 98, 477

Ho, L. C., Filippenko, A. V., \& Sargent, W. L.W. 1997, ApJS, 112, 315

La Franca, F., Fiore, F., Comastri, A., et al. 2005, ApJ, 635, 864

Leahy, D. A., \& Creighton, J. 1993, MNRAS, 263, 314

Luo, B., Bauer, F. E., Brandt, W. N., et al. 2008, ApJS, 179, 19L

Maiolino, R., Salvati, M., Bassani, L., et al. 1998, A\&A, 338, 781

Magdziarz, P., \& Zdziarski, A. A. 1995, MNRAS, 273, 837

Malizia, A., Landi, R., Bassani, L., et al. 2007, ApJ, 668, 81

Marconi, A., Risaliti, G., Gilli, R., et al. 2004, MNRAS, 351, 169

Markwardt, C. B., Barbier, L., Barthelmy, S., et al. 2005, ApJ, 37, 1222

Matt, G., Fabian, A. C., Guainazzi, M., et al. 2000, MNRAS, 318, 173

McElroy, D. B. 1995, ApJS, 100, 105

Nicastro, F. 2000, ApJ, 530, L65

Nicastro, F., Martocchia, A., \& Matt, G. 2003, ApJ, 589, L13

Panessa, F., \& Bassani, L. 2002, A\&A, 394, 435

Panessa, F., Bassani, L., Cappi, M., et al. 2006, A\&A, 455, 173

Pappa, A., Georgantopoulos, I., Stewart, G. C., \& Zezas, A. L. 2001, MNRAS, 326, 995

Risaliti, G., Maiolino, R., \& Salvati, M. 1999, ApJ, 522, 157

Sandage, A., Tammann, G. A., \& Yahil, A. 1979, ApJ, 232, 352

Sazonov, S., Revnivtsev, M., Krivonos, R., Churazov, E., \& Sunyaev, R. 2007, A\&A, 462, 57

Sazonov, S., Revnivtsev, M., Burenin, R., et al. S. 2008, A\&A, 487, 509

Strüder, L., Briel, U., Dennerl, K., et al. 2001, A\&A, 365, L18

Tozzi, P., Gilli, R., Mainieri, V., et al. 2006, A\&A, 451, 457

Treister, E., Urry, C. M., \& Virani, S. 2009, ApJ, 696, 110

Tueller, J., Mushotzky, R. F., Barthelmy, S., et al. 2008, ApJ, 681, 113

Turner, M. J. L., Abbey, A., Arnaud, M., et al. 2001, A\&A, 365, L27

Ubertini, P., Lebrun, F., Di Cocco, G., et al. 2003, A\&A, 411, L131

Ueda, Y., Eguchi, S., Terashima, Y., et al. 2007, ApJ, 664, 79

Vignati, P., Molendi, S., Matt, G., et al. 1999, A\&A, 349, 57

Weaver, K. A., Nousek, J., Yaqoob, T., et al. 1996, ApJ, 458, 160

Winter, L. M., Mushotzky, R. F., Tueller, J., \& Markwardt C. 2008, ApJ, 674, 686

Wolf, C., Wisotzki, L., Borch, A., et al. 2003, A\&A, 408, 499 